\title{
The Chung-Li VHF Radar As a Meteor Radar: System Phase Bias Estimation and Experimental Results
}

\author{
Ruey-Ming Kuong ${ }^{1}$, Yen-Hsyang $\mathrm{Chu}^{2,{ }^{*}}$, Shin-Yi Su${ }^{2}$, and Chung-Lun $\mathrm{Su}^{2}$ \\ (Manuscript received 11 June 2002, in final form 20 February 2003)
}

\begin{abstract}
A new scheme of processing radar returns was developed at the ChungLi VHF radar to estimate the radar system phase bias that is inherently induced by the phase imbalance between receiving channels and hard to determine using conventional methods. With the help of IGRF95 model, echoes from $3 \mathrm{~m}$ field-aligned ionosphere sporadic $\mathrm{E}(\mathrm{Es})$ irregularities have been used successfully to estimate system phase bias of the ionospheric ar. ray of the Chung-Li VHF radar. In an analogous manner, the system phase bias of the interferometer array designed to detect meteor echoes is also determined by comparing observed and expected echoing region of Es fieldaligned irregularities. The results show that the system phase bias is about $60^{\circ}$ between receiving channel pair 1 and 2 , and is about $39^{\circ}$ between receiving channel pair 3 and 2 . After removing the system phase biases, correct angle of arrival of the meteor echoes detected by interferometer array of the Chung-Li VHF radar can be determined unambiguously. The experimental result shows that most meteor echoes distribute within the region illuminated by the main lobe of the antenna beam, not from the sidelobe. Plasma diffusion coefficient and tidal wind in the mesosphere are also measured from radar returns generated from underdense meteor trails associated with the Leonid Meteor Shower during the period from November 13-19, 1997. We find that the height variation of the diffusion coefficient $D$ follows the expression $h=78.84+8.59(\operatorname{lnD})$, very similar to those obtained by other investigators. The mesospheric wind over the Chung-Li radar site is found to be dominated by diurnal tide superimposed by weak semi-diurnal tide. Analysis indicates that the tidal mode responsible for the diurnal tide seems to be $S_{1}^{1}$ mode.
\end{abstract}

(Key words: Sporadic E(Es), Leonid meteor shower,Plasma diffusion coefficient, Tidal wind, System phase bias, Interferometry array, Doppler velocity, Angle of arrival)

\footnotetext{
${ }^{1}$ Chung-Shan Institute of Science and Technology, Long-Tang, Taiwan, ROC

${ }^{2}$ Institute of Space Science, National Central University, Chung-Li, Taiwan, ROC

${ }^{*}$ Corresponding author address: Prof. Yen-Hsyang Chu, Institute of Space Science, National Central University, Chung-Li, Taiwan, ROC; E-mail: yhchu@jupiter.ss.ncu.edu.tw
} 


\section{INTRODUCTION}

The Chung-Li VHF radar consists of three independent and identical modules, and each of the modules contains its own transmitter, receiver, signal processor, antenna array, and other essential system units (Rottger et al. 1990). With this disposition, the Chung-Li VHF radar has the capability of measuring angle of arrival of the echoes from discrete targets in the echoing region by using interferometry technique. In fact, spatial interferometry technique employed for ionospheric investigation was first proposed by Woodman (1971). Subsequently, Farley et al. (1981) modified the interferometry technique by analyzing radar returns in frequency domain. On the basis of this technique, the ionospheric electron density irregularities aligned along magnetic field lines over the Chung-Li radar are observed and investigated. For example, the brightness distribution and aspect angle of nighttime Es echoes from type-2 irregularities were measured (Huang et al. 1995), the nature of quasi-periodic (QP) Es echoes associated with isolated plasma patches occurred periodically in the echoing region was investigated (Chu and Wang 1997; Pan et al. 1998), the spatial structure and Doppler velocities of type-1 and type-2 irregularities were observed (Huang and Chu 1998; Chu and Wang 2002), the properties of a new type of Es radar spectra characterized by low Doppler velocity and extremely narrow spectral width were explored (Chu and Wang 1999), and the drift velocity and three dimensional spatial structure of blob-like Es irregularities are estimated and deduced (Wang and Chu 2001). In addition to ionospheric research, the wind velocity and characteristics of atmospheric refractivity irregularities in lower atmosphere were also measured and investigated with the interferometry method (Pan and Liu 1992; Liu and Pan 1993).

The key factor in using the interferometer method to sense remotely atmosphere is the phase difference of the echoes between a pair of receiving channels. Practically, every radar system has inherent phase bias. Consequently, the estimation of angle of arrival of the target will be incorrect if the system phase bias is not removed from the observed radar returns. In light of the importance of the determination of system phase bias, the algorithms of calibrating system phase bias have been proposed by a number of scientists. For example, the so-called self-survey method was proposed by Valentic et al. (1996) to estimate phase error in the meteor radar by receiving signals transmitted from a given source with known location. In this method, the frequency synthesizer with a small horn antenna was set up on a van and transported to nearby mountain peak for transmission. The exact physical locations selected were determined using a global positioning system (GPS). This kind of method has been applied to the radar located in areas of Søndre Strømfjord, Greenland, and the system phase biases of the radar were estimated. However, because of lacking suitable mountain peaks nearby Chung-Li, this method cannot be applied to the Chung-Li radar. The other method proposed by Palmer et al. (1996) is the radio star method, in which the radio star Cygnus is employed as a given source for the purpose of system phase calibration. This method has been successfully implemented at Japanese MU radar to calibrate its system phase. The limitation of the radio star method is that high antenna gain is required to achieve sufficiently strong signal from radio star for the calibration of system phase. The radio star method is difficult to apply to the interferometer array of the Chung-Li VHF radar because of its low antenna gain and small aperture-power product. 


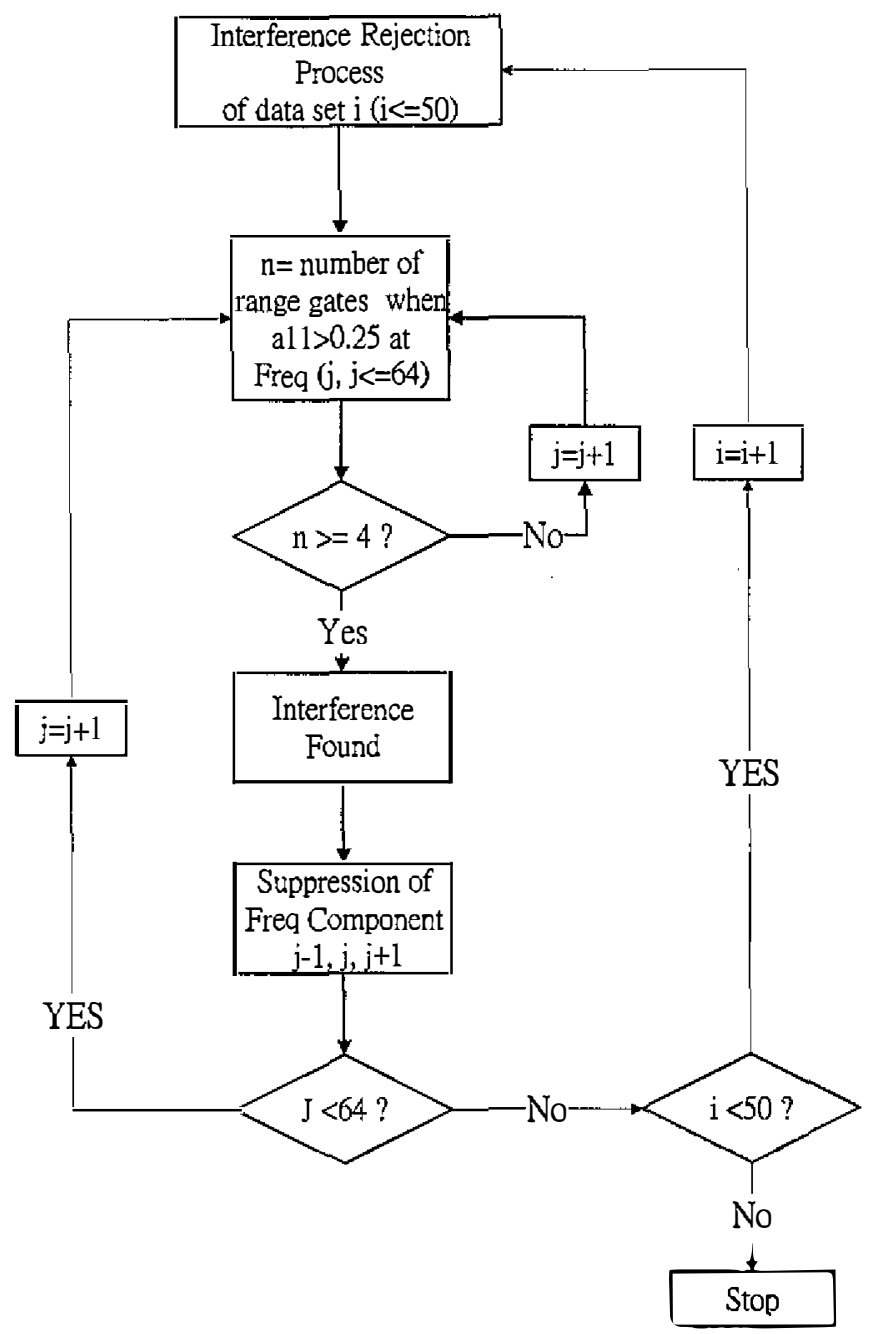

Fig. 4. Flow chart of interference signal rejection .

identification of the desired meteor echoes, the other advantage of this algorithm carried out in frequency domain is the higher sensitivity of rue echo recognition than that in time domain. Notice that one of the required conditions in the use of the interferometry technique to analyze echoes from discrete targets is that the space between adjacent antenna elements in interferometer array has to be close enough to avoid phase ambiguity problem. Also, the broad antenna beam is needed such that the discrete targets located at the places far from the antenna beam axis can be effectively detected and positioned. Consequently, the corresponding antenna gain will be very low so that the weak atmospheric echoes with low signal-to-noise ratio, such as the echoes from meteor trails and Es irregularities in the ionosphere sporadic E layer, will be difficult to distinguish from the intense interference signal in time domain. However, the atmospheric echoes and the interference signals can be separated effectively from each 


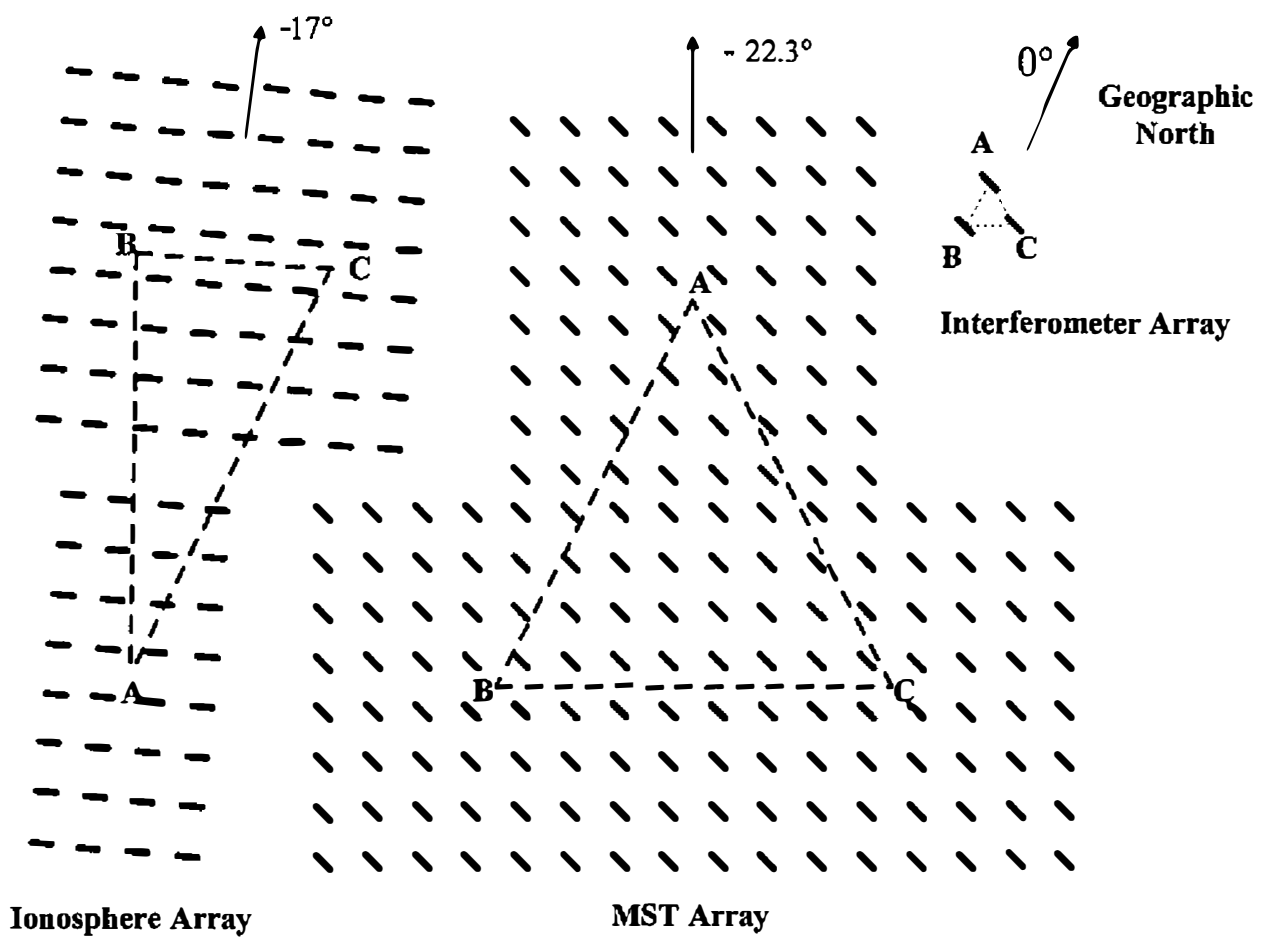

Fig. 1. Antenna configuration of the Chung-Li VHF radar

located on the left-hand side of the MST array is called the ionosphere array that is used to observe the plasma irregularities in the ionosphere. This sub-array consists of three rectangular antenna modules with 32 Yagi elements $(8 \times 4)$ each and is arranged as a triangle with side lengths of $19.6,41.1$, and $44.5 \mathrm{~m}$, respectively. The half power beam width for each antenna module of the ionospheric sub-array is $7.4^{\circ}$ in vertical and $15^{\circ}$ in azimuthal plane. The boresight of the transmitted radar beam is steered toward north by west $17^{\circ}$ geographically with fixed zenith angle $41^{\circ}$ such that the beam axis is perpendicular to the geomagnetic field line at the height of $250 \mathrm{~km}$. However, calculation shows that the zenith angle perpendicular to the field line at the height of $105 \mathrm{~km}$ over Chung-Li radar site is about $38.5^{\circ}$. The smallest sub-array composed of four Yagi antenna elements and located at the upper right corner of the MST array is employed to detect the echoes from meteor trail and is called the meteor array (or interferometer array). Note that during the phase calibration experiment, the radar wave is transmitted by the ionosphere array and received alternately by the ionosphere array and the meteor array through a high-speed RF switch. The three Yagi antennas of interferometer array are arranged in a delta shape with the base line of $5 \mathrm{~m}$ in length, slightly shorter than the radar wavelength of $5.77 \mathrm{~m}$. The phase differences of the returned signals between different pairs of the three Yagi antennas are used to determine the arrival angles of meteor echoes in zenith and azimuth directions. In order to enhance the backscatter and also increase the range resolution of the echoes, the 7-bit Barker code is applied to the radar pulse. Once the radar returns are 
received, the cross spectra of the echoes for each pair of the Yagi antennas are computed. The phase difference of the received echoes between any pair of the Yagi antennas of the interferometer array can thus be deduced by measuring the mean phase around the peak of the coherence (or amplitude) of the cross spectrum. The phase ambiguity problem can be neglected in the interferometer process for meteor array in the observation of Es echoes.

\section{ECHO EXTRACTION PROCEDURE}

The data employed for the current investigations were taken by the Chung-Li VHF radar during the periods from April 21 to 24 and from November 13 to 22, 1997. The procedure of radar signal processing for the Chung-Li VHF radar is presented in the system block as shown in Fig. 2. The echo signals received from the MST array or the ionosphere array pass through high power T/R (Transmitter/Receiver) switch first, and then enter into LNA (Low Noise Amplifier) for amplification. A high-speed RF switch is implemented to select alternately input signals either from the output of LNA or directly from the output of Interferometer array at a rate of pulse repetition frequency. This switch is required when the Es echoes received both by ionosphere and interferometer arrays are recorded for the purpose of system phase

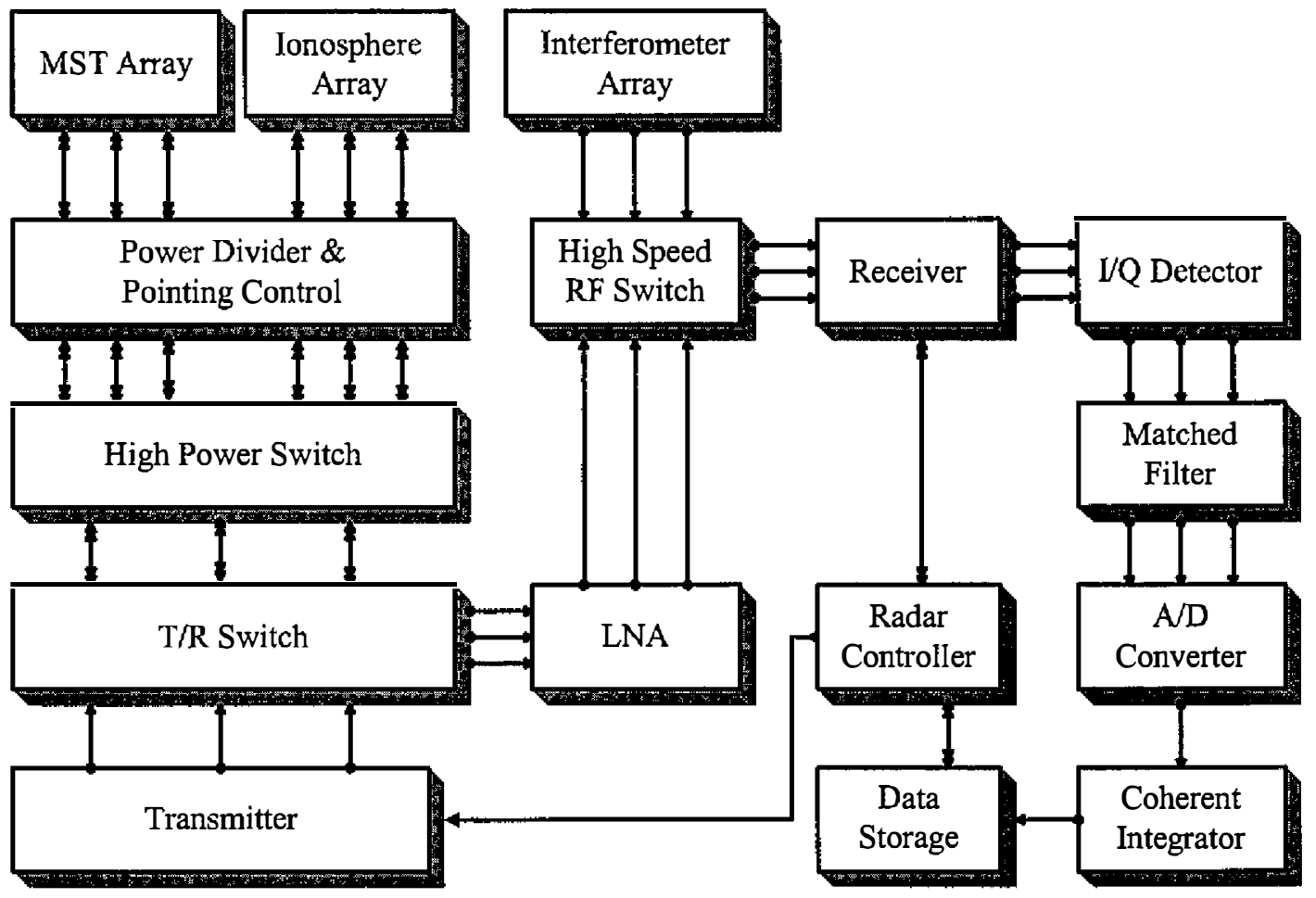

Fig. 2. System block of the Chung-Li VHF radar. 
calibration. The strong Es echoes were detected on April 23 and lasted to early morning on April 24. The collected echoes were transformed into power spectra and subjected to further analysis in frequency domain. We find that the serious interferences from other radio sources may exist in the radar returns detected by interferometer array due to its relatively broad beam width. According to our experiences, the processing of the echoes from meteor trails and Es irregularities in frequency domain will have much higher sensitivity of meteor echo recognition than that in the time domain. Furthermore, an additional advantage of the signal process performed in frequency domain is that the interference signal can be effectively suppressed to obtain more realistic and reasonable spectra for interferometer application on the basis of a special data analysis scheme as mentioned below.

The procedures of identifying the echoes from meteor trails and Es irregularities in frequency domain are shown in Fig. 3. As indicated, the received echoes are first decoded from 7-bit Barker coded signals and compressed into a single range bin. The compressed data is then re-organized and spectrally analyzed by using the 64-point FFT algorithm. The resultant auto-spectrum and cross-spectrum are obtained after four raw spectra were integrated incoherently. Initially, each file of raw data consists of a large amount of non-processed echoes, specifically, 12800 trials per file was set-up in this experiment. Each trial is a complete data set containing echo signals from all range gates and all receive channels. The raw data in each file is further divided into 50 groups with 256 trials each. Every group of data will generate power spectra for every range gate and channel. The data sets X11, X22 and X33 and a11, a22 and a33 represent, respectively, the auto-spectra for three receiving channels before and after normalization. The normalization of the power spectrum was performed with respect to maximum power intensity in the spectrum. The interference signals contained in the normalized power spectrum were then identified and suppressed by a specific interference rejection algorithm. The procedure of this algorithm is shown in Fig. 4. The basic idea of the algorithm is that we first examine frequency by frequency the amplitude of normalized auto-spectra for all range gates. If a specific auto-spectral component with amplitude greater than 0.25 appears simultaneously in four or more successive range gates, this frequency component will be considered a possible interference signal. Once the interference signal is identified in frequency domain, the amplitudes of the original interference signal and two adjacent frequency components are suppressed artificially down to $1 / 1000$ times of the original values. After all frequency components were examined, the true power spectra of the echoes were generated to estimate the angles of arrival of the discrete targets in the scattering volume.

\section{PHASE BIAS ESTIMATION}

A typical example showing the spectra of contaminated meteor echoes before and after interference rejection is displayed in Fig. 5. The top, middle, and bottom panels on the left hand side of the figure represent, respectively, the range-frequency auto-spectrogram, normalized cross-spectrum at range gate 52 , and the corresponding phase spectrum before the procedure of suppressing interference signal. The panels on the right side of Fig. 5 are the spectra after interference suppression. As shown, the exceedingly large spectral peaks in the auto- and 


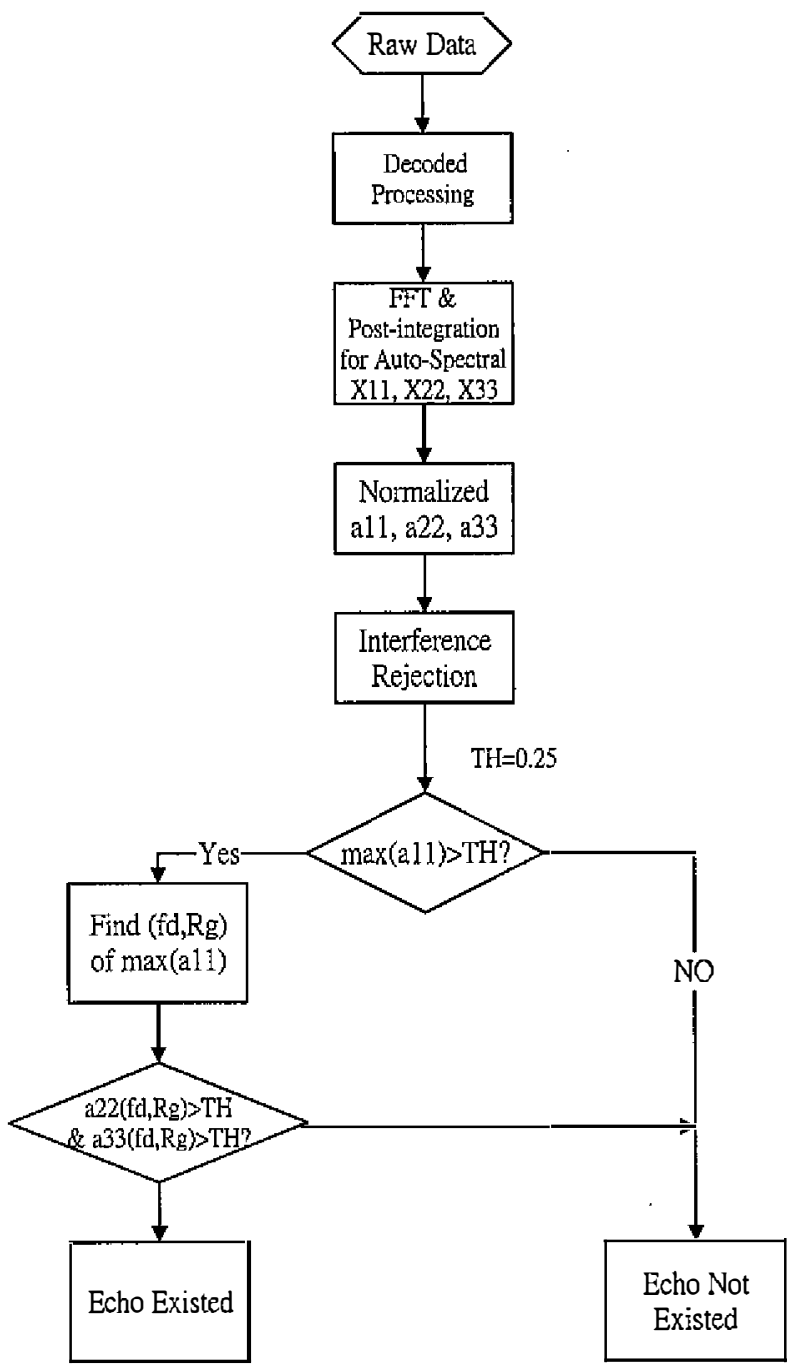

Fig. 3. Flow chart of signal processing for the identification of meteor echoes in frequency domain.

cross-spectra at frequency around $51 \mathrm{~Hz}$ are interference signals. Obviously, interference signals not only dominate the original auto- and cross-spectra, but also distort the corresponding phase spectrum, producing incorrect phase information for the use of spatial interferometer. However, desired power spectra and phase information can be obtained after the procedure of interference rejection is performed. This example clearly shows that the above algorithm can extract effectively true echoes from the contaminated echoes. In light of the fact that the behavior of amplitude (or power) and phase spectra of the atmospheric echoes will be intervened significantly by the interference signal, identification and removal of the interference signal from observed radar returns is essential to the radar signal processing. Except for the effective 


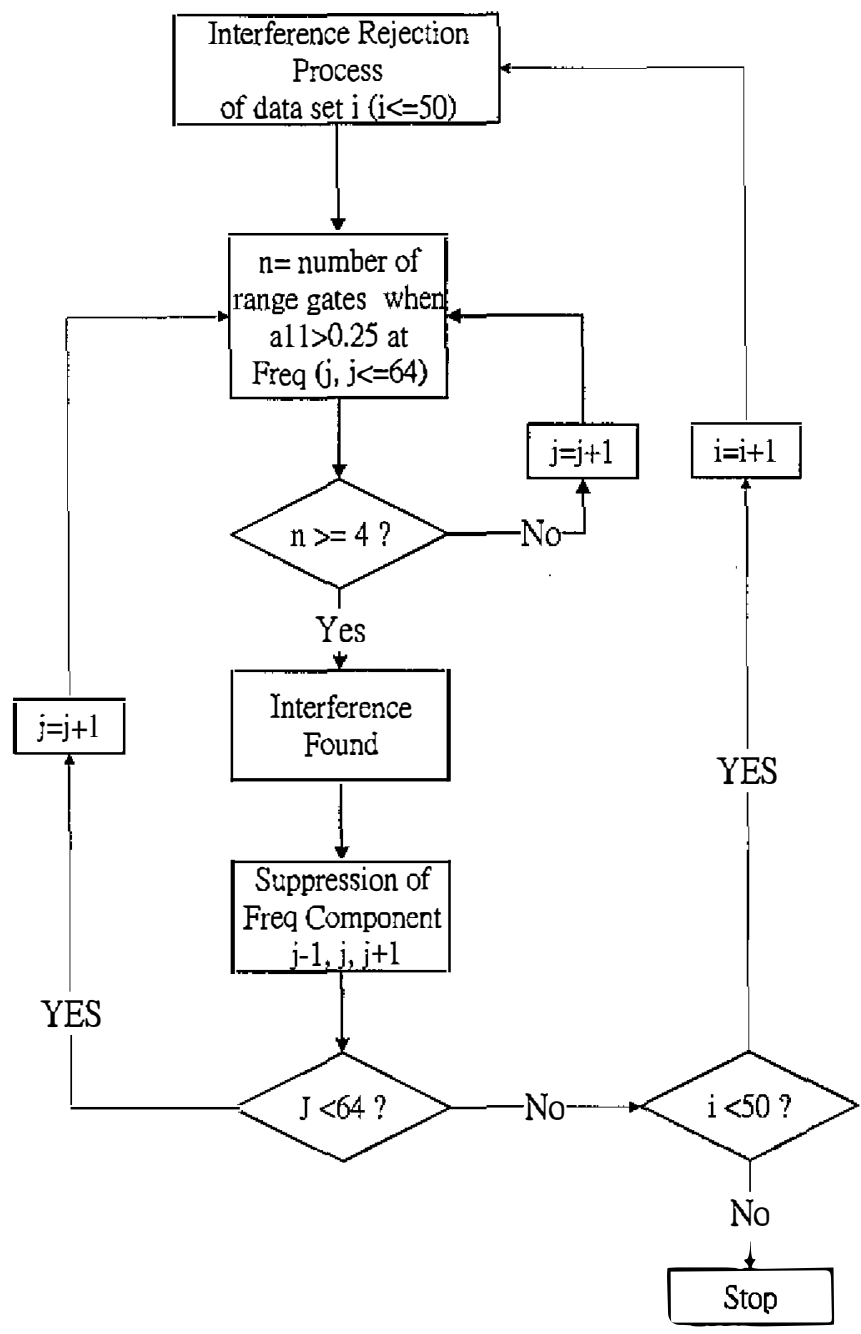

Fig. 4. Flow chart of interference signal rejection .

identification of the desired meteor echoes, the other advantage of this algorithm carried out in frequency domain is the higher sensitivity of rue echo recognition than that in time domain. Notice that one of the required conditions in the use of the interferometry technique to analyze echoes from discrete targets is that the space between adjacent antenna elements in interferometer array has to be close enough to avoid phase ambiguity problem. Also, the broad antenna beam is needed such that the discrete targets located at the places far from the antenna beam axis can be effectively detected and positioned. Consequently, the corresponding antenna gain will be very low so that the weak atmospheric echoes with low signal-to-noise ratio, such as the echoes from meteor trails and Es irregularities in the ionosphere sporadic E layer, will be difficult to distinguish from the intense interference signal in time domain. However, the atmospheric echoes and the interference signals can be separated effectively from each 

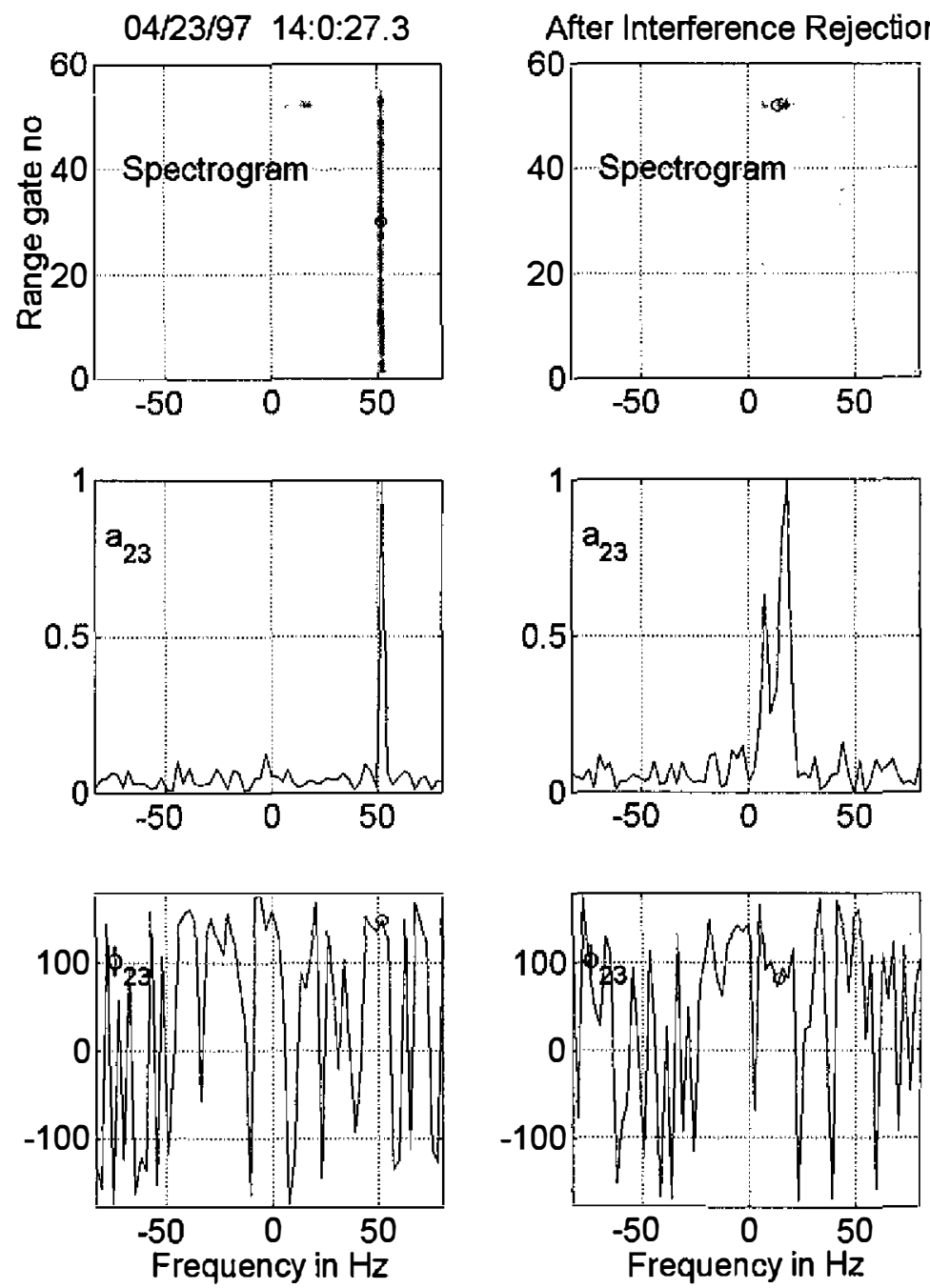

Fig. 5. Range gate variation of auto-spectra, normalized cross-spectrum and the corresponding phase spectrum before (panels on the left side) and after (panels on the right side) interference signal rejection.

other in frequency domain. This is because most of the interference signals around the ChungLi radar site are characterized by narrow-band signals with relatively stable and constant carrier frequencies compared to the atmospheric echoes. The panels on the left hand side of Fig. 6 present examples of noise-like echoes signals in time domain, in which the top panel is the range-time-intensity contour plot, the middle and bottom panels are, respectively, the time series of echo intensity and phase at range gate with maximum intensity. As indicated, the noise and true signals cannot be distinguished clearly from each other in time domain. However, the true signal embedded in noise-like echoes can be clearly seen in frequency domain as shown in panels in the right hand side of Fig. 6. Therefore, by using the algorithm of interfer- 

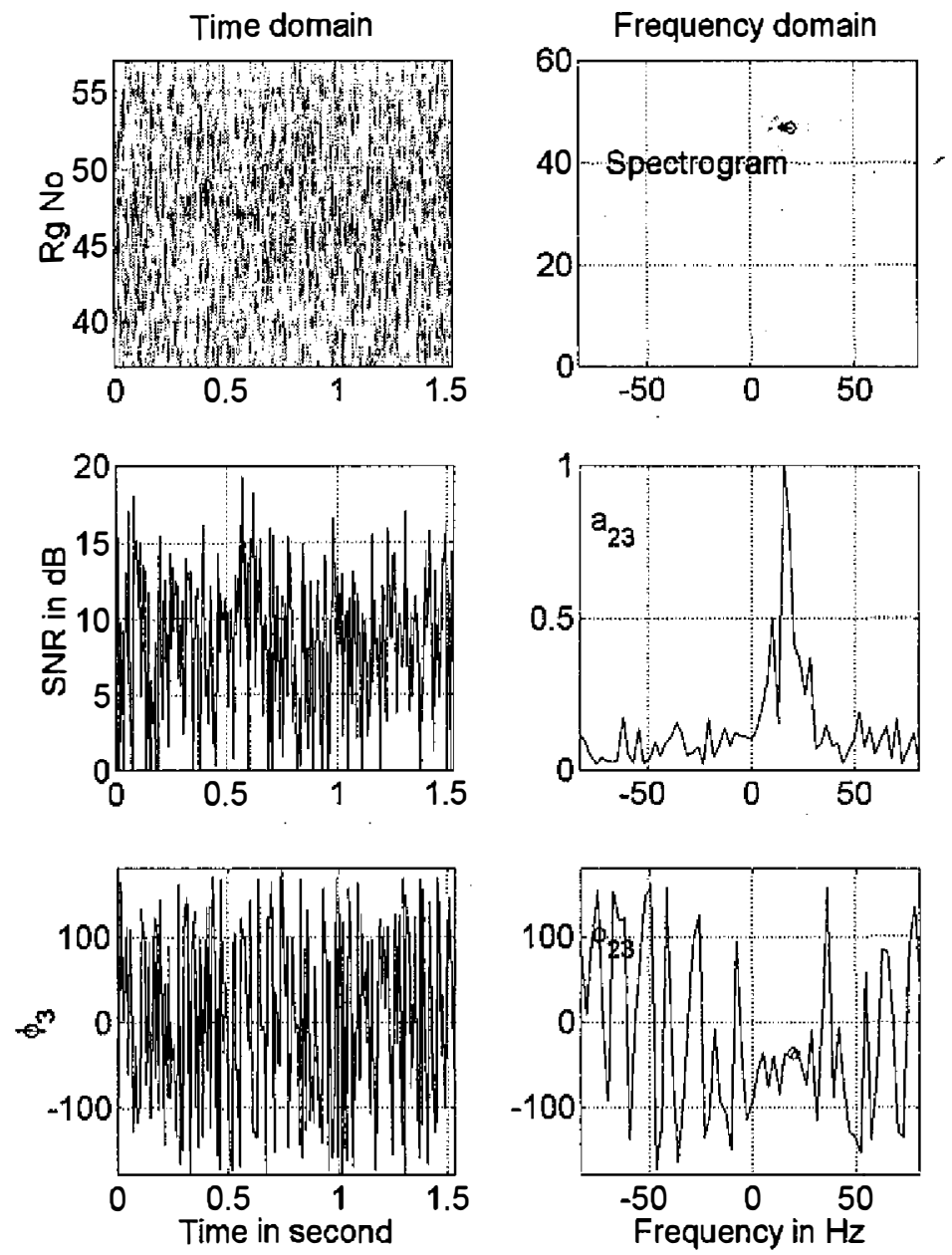

Fig. 6. Examples of noise-like echoes signals in time domain before (panels in left side) and after (panels in right side) interference signal rejection, in which top panel is range-time-intensity contour plot, the middle and bottom panels are, respectively, the time series of echo intensity and phase at range gate with maximum intensity.

ence rejection as mentioned above, the true spectra and correct phase information of the echo signals can thus be obtained.

Both ionosphere array and interferometer array of the Chung-Li VHF radar can detect meteor and Es echoes after suitable radar parameters are set. With the help of Es echoes received by ionosphere array, which has been well calibrated, the system phase bias of interferometer array can be determined. According to Wang (1999) and Wang and Chu (2001), the system phase bias of ionosphere array was estimated statistically by comparing observed and expected echoing regions calculated by using IGRF95. It shows that the expected echoing region for $3 \mathrm{~m}$ field-aligned Es irregularities detected by the Chung-Li VHF radar is locally 
confined within a specific region with angular extents of about $38^{\circ}-41^{\circ}$ in zenith and $-15^{\circ}-5^{\circ}$ in azimuth directions, in which the minus sign for azimuth angle indicates the angle to the west side with respect to the boresight of antenna beam. As a result, the system phase bias can be estimated by comparing observed and expected echoing regions after a large amount of Es data are collected and analyzed.

The phase calibration experiment for the interferometer array was conducted by operating switching mode, namely, the radar echoes received by ionosphere and interferometer arrays are recorded alternately pulse by pulse through high speed RF switch. Typical range-timeintensity contour plots of Es echoes received by two antenna arrays are presented in Fig. 7. As shown, in general, the echo intensity from ionosphere array is about twice stronger than that from interferometer array due to antenna gain difference in the two. Once the phase information of the echoes is obtained, the angle of arrival of the target can be calculated directly in accordance with a set of proper interferometer equations. The interferometry equations employed for the calculation of arrival of angle of meteor echoes for the Chung-Li VHF radar are derived below. Let the azimuth angle $\phi$ be positive while measuring the angle clockwise from a direction of $22.3^{\circ}$ north by west and the zenith angle $\theta$ be positive while measuring the angle with respect to the vertical axis. According to the configuration of interferometry array as shown in Fig. 1, $\theta$ and $\phi$ can be computed in accordance with the following equations

$$
\begin{aligned}
& \phi=\tan ^{-1}\left(\frac{\frac{\sqrt{3}}{2} \varphi_{32}}{\varphi_{12}-0.5 \varphi_{32}}\right)+\Delta \phi \\
& \theta=\sin ^{-1}\left(\frac{\varphi_{32}}{k d \sin \phi}\right)
\end{aligned}
$$

where $\mathrm{k}$ is wavenumber $(k=2 \pi / \lambda, \lambda$ is radar wavelength); $\mathrm{d}(=5 \mathrm{~m})$ is the distance between adjacent interferometer antennas, $\varphi_{12}$ is the phase difference between antenna pair 2 and 1 $\left(\varphi_{12}=\varphi_{2}-\varphi_{1}\right)$, and $\varphi_{32}$ is the phase difference between antenna pair 2 and $3\left(\varphi_{32}=\varphi_{2}-\varphi_{3}\right)$. Note that the value of $\Delta \phi$ may be $0, \pi$ or $-\pi$, depending on the sign of numerator and denominator in Eq.(1). Namely, $\Delta \phi$ should be set to be zero when denominator is positive, $\Delta \phi$ should be $\pi$ when the denominator is negative and numerator is positive, while $\Delta \phi$ will be set to be $-\pi$ when denominator and the numerator are both negative. For the special case of $\varphi_{32}=0$, we have $\phi=0$ and $\theta=\sin ^{-1}\left[\varphi_{12} /(k d \sqrt{3} / 2)\right]$. The observed phase differences of Es echoes between a pair of receiving channels can be obtained directly from the phase information around spectral peak of cross-spectrum as shown in Fig. 8, in which the spectral peak with coherence greater than 0.7 are adopted for analysis. The scatter points marked with open circle and dot in Fig. 8 are, respectively, the data before and after system phase bias adjustment. The area surrounded by solid lines represents the region of expected phase differences and angles of arrival for the radar returns from Es irregularities centered at the height of $100 \mathrm{~km}$ with 

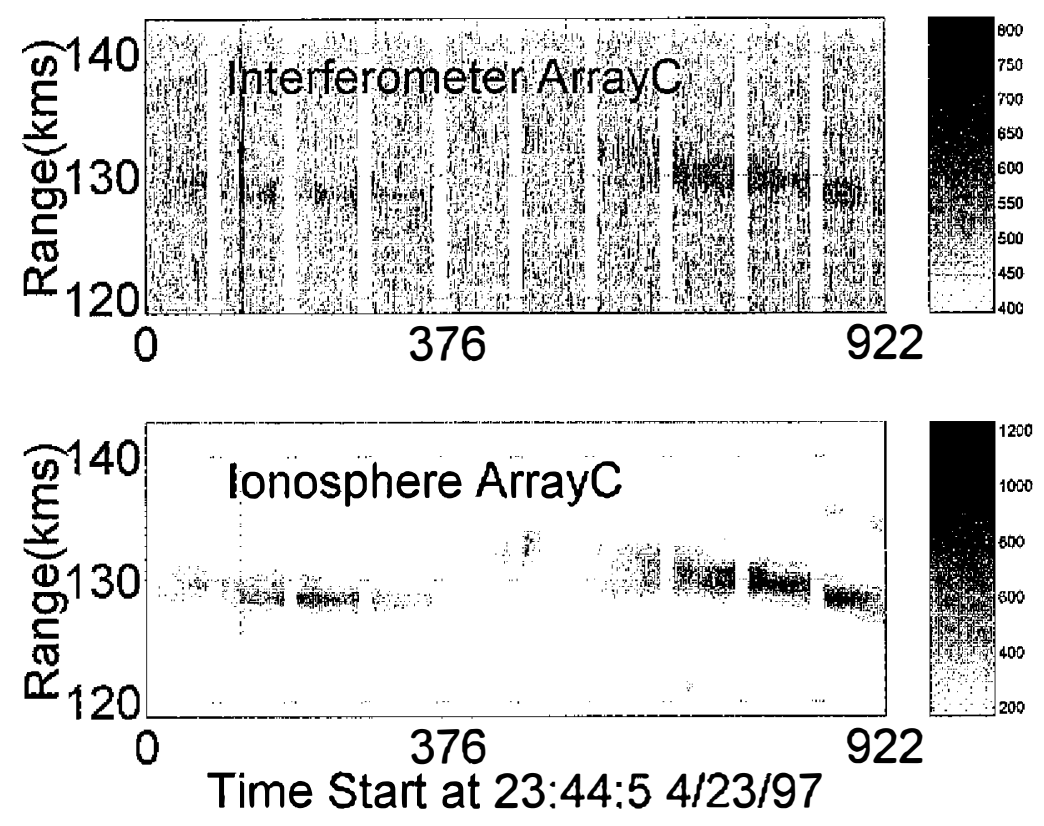

Fig. 7. Range-time-intensity plots of echoes from Es irregularities, in which lower and upper panels are, respectively, the Es echoes received by ionosphere and interferometer arrays.

vertical extent of $4 \mathrm{~km}$. Therefore, from Fig. 8 we estimate that the system phase biases of the interferometer array in $\varphi_{32}$ and $\varphi_{12}$ are, respectively, about $39^{\circ}$ and $60^{\circ}$. To verify the correctness of estimated system phase bias, the spatial distribution of meteor echoes obtained during the experiment is shown in Fig. 9. It is obvious from Fig. 9 that most of the meteor echoes locate primarily within the main beam of ionosphere antenna array. Once the system phase bias of interferometer array is adjusted, the exact altitude and angle of arrival of the meteor echoes can thus be determined accurately in accordance with Eqs. (1) and (2) combined with range information.

\section{OBSERVATIONS}

In this section, the observational results of mesospheric diffusion coefficient and winds and tides in the height range between 80 and $100 \mathrm{~km}$ are presented. The meteor data were taken during the period of Leonid Meteor Shower from November 13 to 19, 1997. The radar parameters were set as follows: transmitted peak power of $75 \mathrm{~kW}$, inter-pulse period of $3 \mathrm{~ms}$, pulse width of $52 \mu$ s with 13-baud Barker code (corresponding to range resolution of $600 \mathrm{~m}$ ), delay time of $480 \mu \mathrm{s}$, coherent integration time of $12 \mathrm{~ms}$, and 120 range gates were set. In addition, in order to observe mesospheric winds, three antenna beams of MST array were steered simultaneously toward vertical, north, and east directions. The meteor echoes were detected by interferometer array and discriminated in accordance with the signal processing 

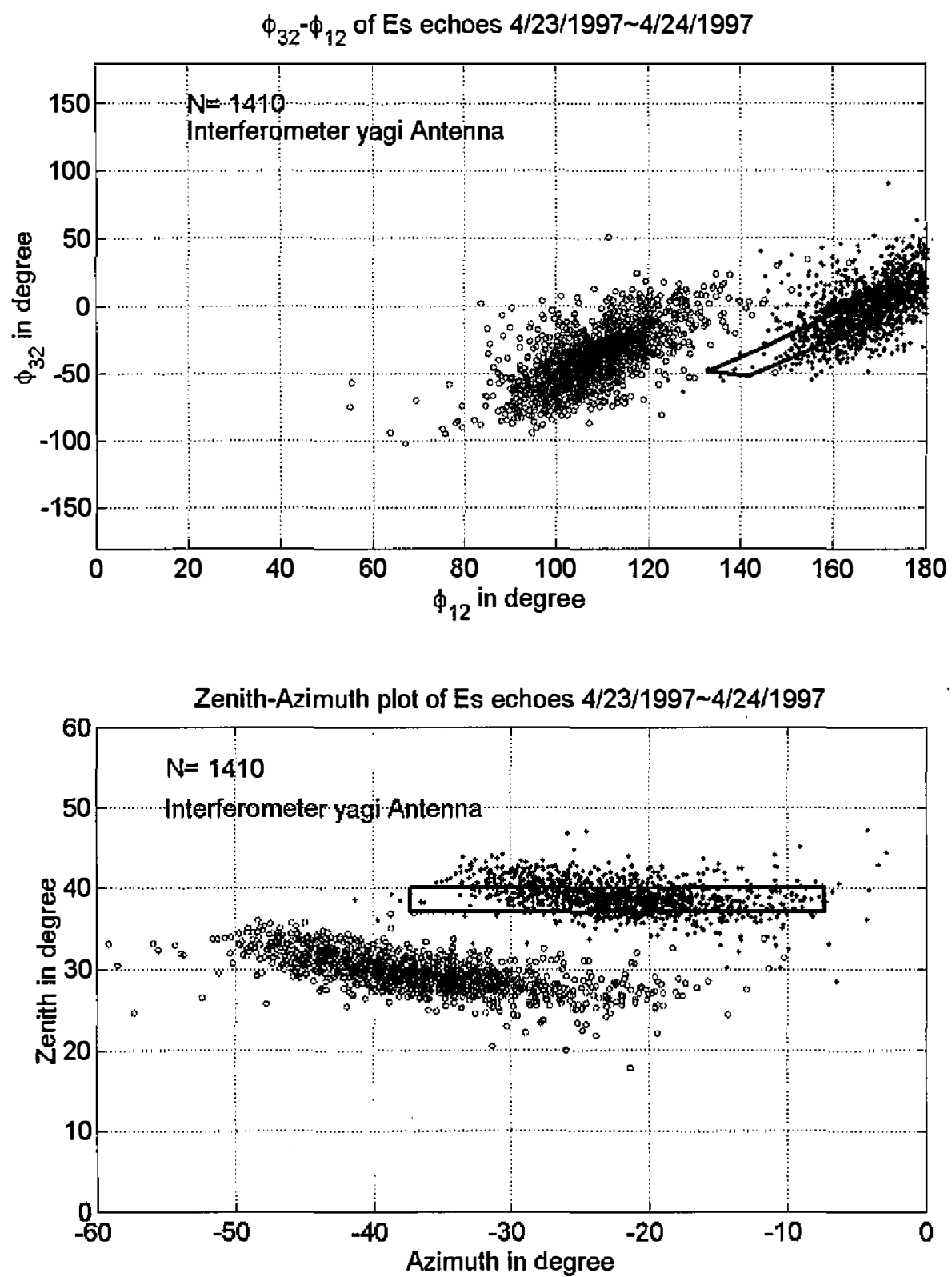

Fig. 8. Scatter diagrams showing $\varphi_{32}$ versus $\varphi_{12}$ and $\theta$ versus $\phi$ determined by using Interferometer array, respectively. Note that the points marked with open circle and dot are, respectively, the data before and after system phase bias adjustment. The rectangle circling the center of the group with dots represents predicted echoing region corresponding to the Es irregularities centered at $100 \mathrm{~km}$ with vertical extent of $4 \mathrm{~km}$. 


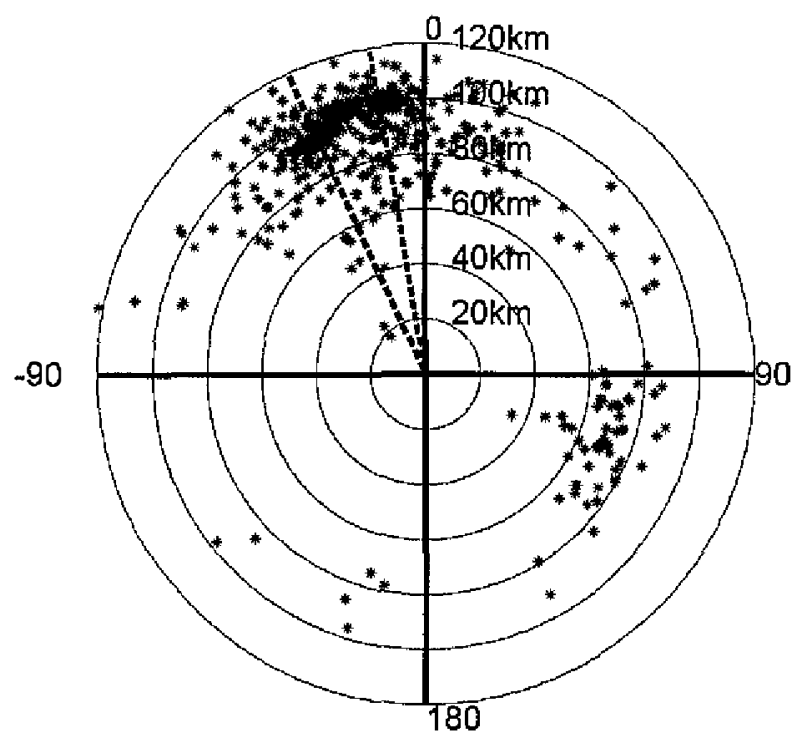

Fig. 9. Scatter diagram showing the height and azimuth direction of the meteor echoes detected by ionosphere array, in which two dashed lines represent the primary illuminating region of the antenna beam. Note that the boresight of antenna beam of the ionosphere array is steered toward north by west $22.3^{\circ}$.

scheme as mentioned earlier.

The mesospheric diffusion coefficient $\mathrm{D}$ deduced from the fading of the echo power of the meteor trail can be estimated in accordance with following expression (McKinley 1961)

$$
D=\frac{\lambda^{2}}{32 \pi^{2} \tau},
$$

where $\tau$ is the time that the intensity of radar returns from underdense meteor trail decays to $\mathrm{e}^{-1}$, and $\lambda$ is radar wavelength. It is noteworthy that $\mathrm{D}$ is a strong function of height, because the collision between ion and neutral dominating the behavior of $\mathrm{D}$ is highly altitude-dependent. Therefore, accurate altitude estimation of the meteor trail is required to obtain correct height variation of diffusion coefficient. Assume that $\mathrm{D}$ increases with height exponentially, namely, $\mathrm{D}=\mathrm{D}_{0} \exp \left(\mathrm{h} / \mathrm{H}_{\mathrm{D}}\right)$, where $\mathrm{H}_{\mathrm{D}}$ is the scale height. We have

$$
h=H_{D} \ln D-H_{D} \ln D_{0} .
$$

Therefore, $H_{D}$ and $D_{0}$ can be estimated in accordance with (4) if height variation of D is obtained through the observation of underdense meteor trails. Fig. 10 shows the height variations of $\mathrm{D}$ before (upper panel) and after (lower panel) system phase bias adjustment. As indicated, a significant influence on the behavior of D varied with height is seen. After best fitting (4) to observed data, shown in Fig. 10b, by means of least squares method, we obtain the expression 

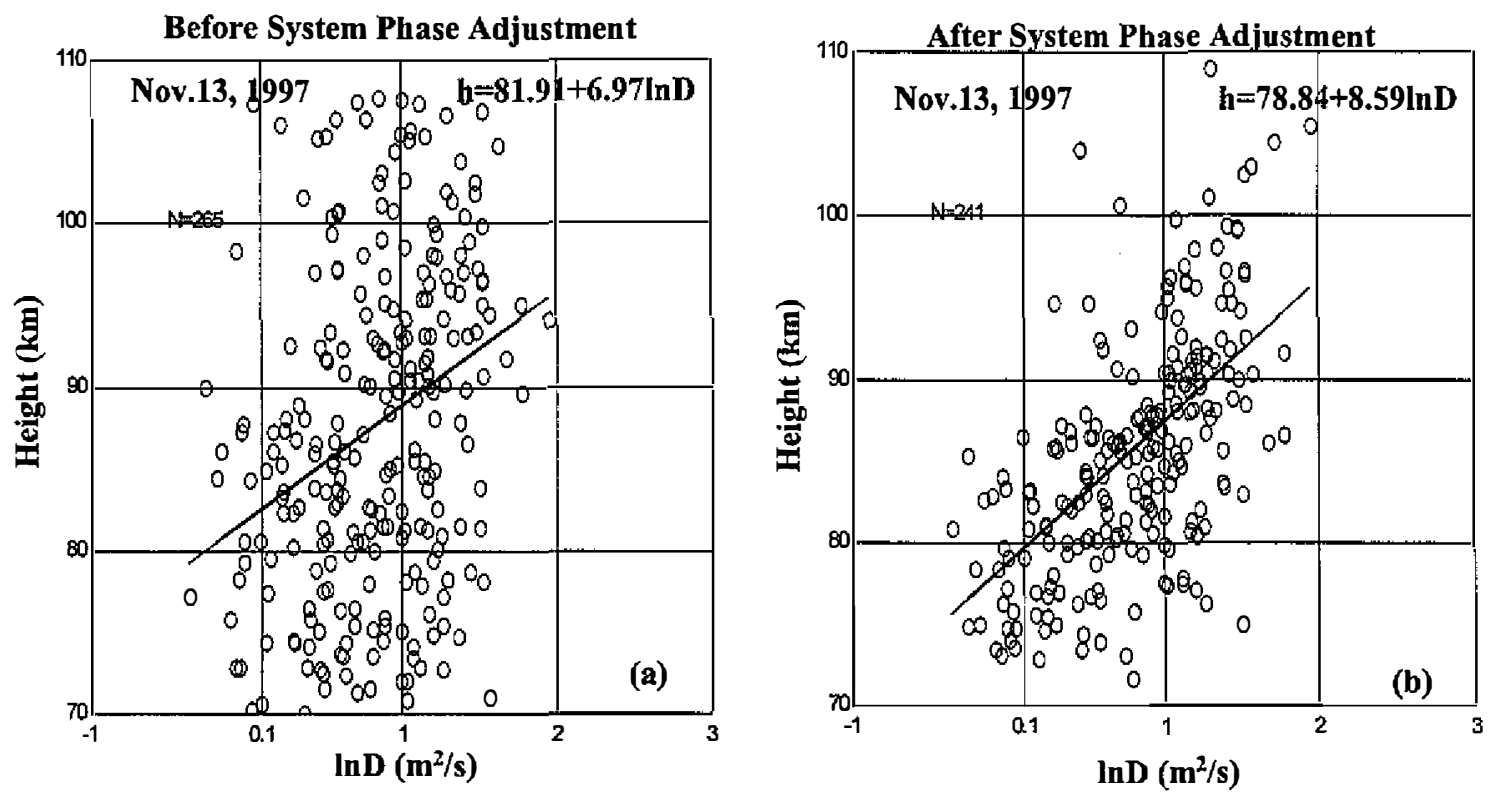

Fig. 10. A comparison of height variations of ambipolar diffusion coefficient before (a) and after (b) system phase bias adjustment.

connecting $\mathrm{D}$ and $\mathrm{h}$ as follows

$$
h=78.84+8.59 \cdot \ln D \text {. }
$$

Therefore, the corresponding $H_{D}$ and $D_{0}$ are, respectively, $8.59 \mathrm{~km}$ and $0.000103 \mathrm{~m}^{2} \mathrm{~s}^{-1}$. Note that our results presented in (4) is very similar to those obtained by Jones (1970) (i.e., 81+7. 4.lnD), Tsutsumi et al. (1994) (i.e., $h=72+8.7 \cdot \ln D$ ) and Nakamura et al. (1991) (i.e., $h=76$. $916+11.533 \cdot \ln D$ ), further validating the applicability of estimated system phase bias as mentioned in previous section.

With the information on Doppler velocity and angle of arrival of meteor echoes, the mesospheric wind and tides can be measured. Assume that the vertical wind in meteor height is so weak that it is able to be ignored (Valentic et al. 1996). As a result, the relation between radial velocity $\mathrm{V}_{\mathrm{r}}$ of meteor trail, horizontal wind velocity $\mathrm{V}$, and angular position of meteor trail in illuminating region can be formulated below

$$
V_{r}=V \cos \left(\phi_{V}-\phi_{M}\right) \sin \theta_{M}
$$

Where $\phi_{\mathrm{M}}$ and $\phi_{\mathrm{V}}$ are, respectively, the azimuth angle of meteor trail and horizontal wind direction with respect to eastward direction, and $\theta_{\mathrm{M}}$ is the zenith angle of meteor trail. If there are $\mathrm{N}$ meteor trails subjected to a constant drift by uniform wind in a relatively short period and narrow height extent, (6) can be expressed as 


$$
\left[\begin{array}{l}
V_{r_{1}} \\
V_{r_{2}} \\
\vdots \\
V_{r_{N}}
\end{array}\right]=\left[\begin{array}{ll}
\cos \phi_{M_{1}} \sin \theta_{M_{1}} & \sin \phi_{M_{1}} \sin \theta_{M_{1}} \\
\cos \phi_{M_{2}} \sin \theta_{M_{2}} & \sin \phi_{M_{2}} \sin \theta_{M_{2}} \\
\vdots &
\end{array}\right]\left[\begin{array}{l}
V \cos \phi_{V} \\
V \sin \phi_{V}
\end{array}\right] .
$$

Note that $V \cos \phi_{\mathrm{V}}$ and $\mathrm{V} \sin \phi_{\mathrm{V}}$ in (7) correspond to zonal $\mathrm{u}$ and meridional $\mathrm{v}$ wind velocities, respectively. In matrix form, (7) can be compactly written as

$$
\mathbf{V r}=\mathbf{A V} \text {, }
$$

where $A$ as the function of $\theta$ and $\phi$ is a Nx2 matrix from $\mathrm{N}$ observations, $\mathrm{V}$ is a matrix with 2 elements, i.e., true velocity components $\mathrm{u}$ and $\mathrm{v}, \mathrm{Vr}$ is a $\mathrm{Nx} 1$ matrix consisting of the Doppler velocities of the targets. Solving for the vector $\mathrm{V}$ in the least-squares sense gives

$$
\mathbf{V}=\left(\mathbf{A}^{\prime} \mathbf{A}\right)^{-\mathbf{1}} \mathbf{A}^{\mathbf{\prime}} \mathbf{V} \mathbf{r},
$$

where $\mathbf{A}^{\prime}$ is the transpose of $\mathbf{A}$. After collecting sufficient data and performing matrix manipulation, $V \cos \phi_{\mathrm{V}}$ and $\mathrm{V} \sin \phi_{\mathrm{V}}$ can be estimated. Figs. 11a and b are, respectively, meridional and zonal components of hourly wind velocities in the height range $80-100 \mathrm{~km}$ for the period of 13-19 November 1997. Notice that a sliding window with time interval of 4 hours and height coverage of 4 kilometers was employed to smooth the irregular fluctuations of the wind velocities estimated from the least squares method. As shown, a striking and stable diurnal variation of wind velocities is seen, especially for the meridional component. The maximum wind velocity is as large as more than $85 \mathrm{~m} \mathrm{~s}^{-1}$ at altitude around $97 \mathrm{~km}$. From Fig. $11 \mathrm{a}$, it appears that the phases in meridional component of diurnal tidal winds are propagating downward and the corresponding vertical wavelength of the diurnal tide is about $30-40 \mathrm{~km}$, suggesting that the tidal mode responsible for the diurnal variation of wind velocity is $S_{1}^{i}$ mode. In addition to the change in phase with height, the amplitude of diurnal tide shows a trend of increase with height and maximum at altitude around $97 \mathrm{~km}$. It is noteworthy that, irrespective of strong diurnal variation in meridional component, a weak but recognizable semi-diurnal variation in zonal wind component superimposes on the diurnal variation of tidal motion in height range between 83 and $94 \mathrm{~km}$. Fig. $11 \mathrm{~b}$ further indicates that semi-diurnal variation in zonal component is not significant and hard to identify in the height regions below $84 \mathrm{~km}$ and above $95 \mathrm{~km}$. In general, semi-diurnal tide can be excited by latent heat release of water vapor in lower atmosphere in tropical and subtropical regions and propagates vertically. Also, ozone in the stratosphere may be another important mechanism responsible for the excitation of propagating semi-diurnal tide. It suggests that change in excitation mechanism will change in the vertical propagation of semi-diurnal tide in the atmosphere. Therefore, the features presented in Fig. 11 imply that, presumably, there are local structures or disturbances in 

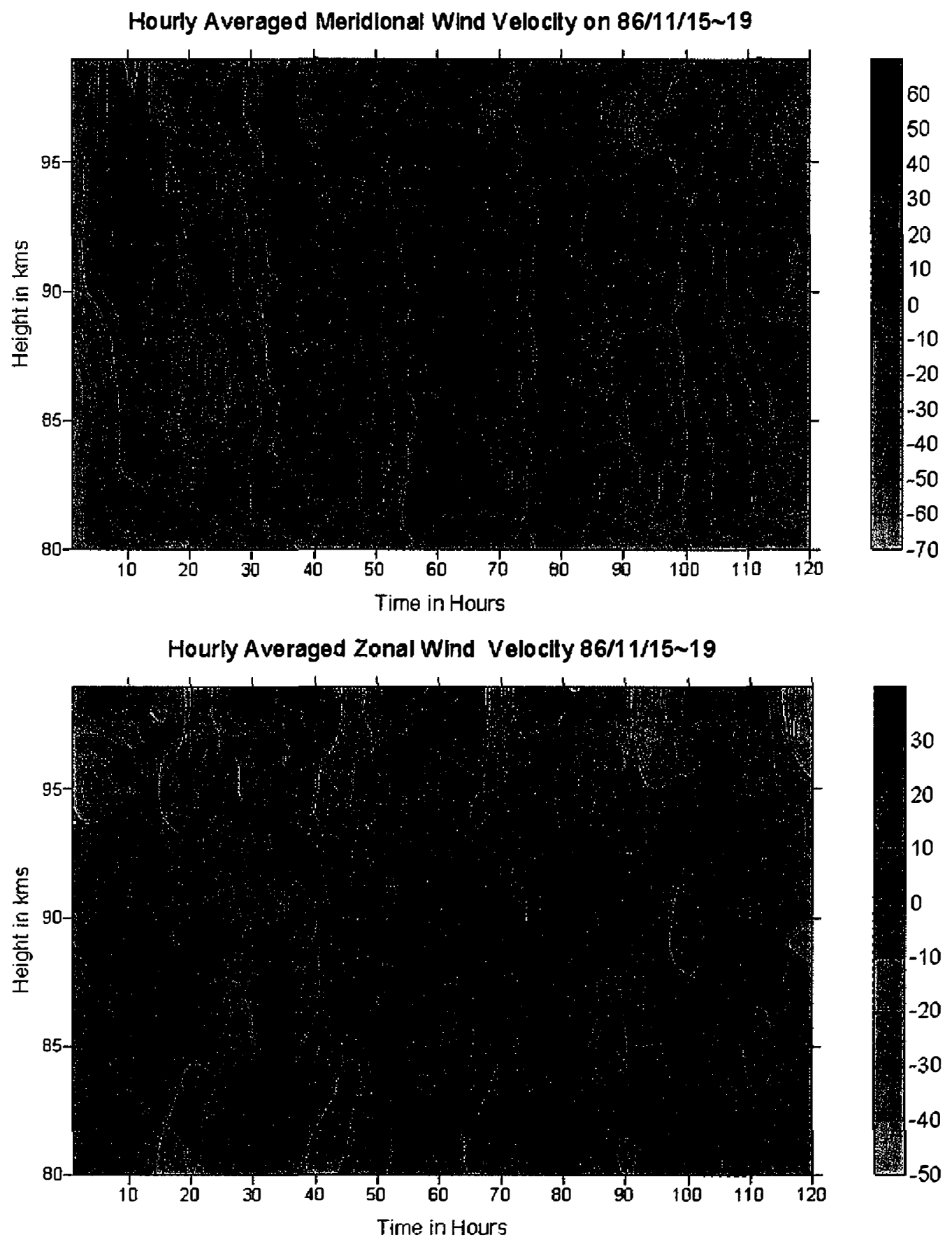

Fig. 11. Height-time variations of hourly wind velocities (in unit of $\mathrm{m} \mathrm{s}^{-1}$ ) in the height range 80-100 km during 1997 Leonid Meteor Shower. (a) Meridional wind. (b) Zonal wind. 
the mesosphere affecting the propagations of semi-diurnal tides vertically. Except for the local effect, other effects, including abnormal propagation of tidal waves in vertical direction, dayto-day variability, non-linear coupling between different modes, and change in the source of tide excitation, may also influence the behavior of tidal waves in mesosphere. It is not easy to explain satisfactorily the cause of observed features of tidal motions in mesosphere only based on the observations made by a single station. Large amount of observational data incorporating theoretical works are required to understand the insight of the tidal wave propagation in the atmosphere.

\section{CONCLUSION}

A new method employed to calibrate the system phase bias of interferometer array of the Chung-Li VHF radar has been established successfully. With the help of a well-calibrated ionosphere array and IGRF95 model, the system phase bias of interferometer array is determined by carefully comparing observed and expected echoing regions. In light of the very low antenna gain of interferometer array, it is inappropriate to discriminate the meteor echoes from other signals embedded in radar returns in time domain, especially in conditions with strong interference signals. A signal processing scheme is developed in this investigation, in which the discrimination of meteor echoes from radar returns contaminated by interference signals is carried out in frequency domain to increase the sensitivity of meteor echo detection and suppress effectively the interference signals in the Doppler spectrum After adjusting the system phase bias, the characteristics of mesospheric diffusion coefficients and mesospheric winds and tides over the Chung-Li radar site are measured from radar returns of underdense meteor trails. It shows that height variation of the diffusion coefficient $\mathrm{D}$ can be described by expression $\mathrm{h}=78.84+8.59(\ln \mathrm{D})$, very similar to those obtained by other investigators. The mesospheric winds in the period of Leonid Meteor Shower from 13-19 November 1997, are observed. We find that a salient and stable diurnal variation dominates the meridional component of mesospheric wind. Analysis indicates that the tidal mode responsible for the diurnal variation seems to be $S_{1}^{1}$ mode. Furthermore, a weak but discernable semi-diurnal variation appears in zonal component of mesospheric wind in the height range $83-94 \mathrm{~km}$. More observations of mesospheric winds and waves deduced from radar returns of underdense meteor trails are needed to ensure that the data described in this investigation are representative of typical behavior of mesospheric wind.

Acknowledgments This work was partially supported by National Science of Taiwan under Grants NSC-89-2111-M-008-046 and NSC-90-NSPO(A)-ECP-008-01.

\section{REFERENCES}

Chu, Y. H., and C. Y. Wang, 1997: Interferometry observations of three-dimensional spatial structures of sporadic E irregularities using the Chung-Li VHF radar. Radio Sci., 32, 817-832. 
Chu, Y. H., and C. Y. Wang, 1999: Interferometry investigations of VHF backscatter from plasma irregularity patches in the nighttime E region using the Chung-Li radar. $J$. Geophys. Res., 104, 2621-2631.

Chu, Y. H., and C. Y.Wang, 2002: Plasma Structures of $3 \mathrm{~m}$ Type 1 and Type 2 Irregularities in Nighttime Mid-latitude Sporadic E Region. J. Geophys. Res. (accepted).

Farley, D. T., H. M. Ierkic, and B. G. Fejer, 1981: Radar Interferometry: A new technique for studying plasma turbulence in the ionosphere. J. Geophys. Res., 86, 1467-1472.

Huang, C. M., E. Kudeki, S. J. Frank, C. H. Liu, and J. Rottger, 1995: Brightness distribution of midlatitude E region echoes detected at Chung-LI VHF radar. J. Geophys. Res., 100, 14703-14715.

Huang, C. M., and Y. H. Chu, 1998: First observation of type-1 sporadic E irregularities in equatorial anomaly region by using Chung-Li VHF radar. Geophys. Res. Lett., 25, 37793782.

Johnes, J., 1970: On the variation of the ambipolar diffusive coefficient with height. Planet. Space Sci., 18, 1836-1842.

Kuong, R. M., 1998: Establishment of system and technical method for the measurements of meteor rail at the Chung-VHF radar, Ph.D. dissertation, National Central University, Taiwan (R.O.C.) (in Chinese).

Liu, C. H., and C. J. Pan, 1993: New observational techniques for studying the dynamics of the middle atmosphere using the Chung-Li VHF radar. J. Atmos. Terr. Phys., 55, 1055 1066.

McKinley, D. W. R., 1961: Meteor sciences and engineering, p.201, McGraw-Hill Book Co., New York.

Nakamura, T., T. Tsuda, and M. Tsutsumi, 1991: Meteor wind observations with the MU radar. Radio Sci., 24, 857-869.

Palmer, R. D., S. Vangal, M. F. Larsen, S. Fukao, T. Nakamura, and M. Yamamoto, 1996: Phase calibration of VHF spatial interferometry radars using stellar sources. Radio Sci., 31, 147-156.

Pan, C. J., and C. H. Liu, 1992: A model for oblique spaced antenna technique for mesosphere-stratosphere-troposphere radars and its applications. Radio Sci., 27, 131-144.

Pan, C. J., C. H. Liu, J. Rottger, S. Y. Su., and J. Y. Liu, 1998: E region observations over Chung-Li during the SEEK campaign. Geophys. Res. Lett., 25, 1805-1808.

Rottger, J., C. H. Liu, J. K. Chao, A. J. Chen, C. J. Pan, and I. J. Fu, 1990: Spatial interferometer measurements with the Chung-Li VHF radar. Radio Sci., 25, 503-515.

Tsutsumi, M., T. Tsuda, and S, Fukao, 1994: Temperature fluctuations near the mesopause inferred from meteor observations with the middle and upper atmosphere radar. Radio Sci., 29, 599-610.

Valentic, T. A., S. K. Avery, and J. P. Avery, 1996: MEDAC/SC: A third generation meteor echo detection and collection system. IEEE Geosci. Remote Sens., 34, 15-21.

Wang, C. Y., 1999: The observations of ionospheric sporadic Eirregularities using the ChungLi VHF radar, Ph.D dissertation, National Central University, Chung-Li, Taiwan (R.O. C.) (in Chinese).

Wang, C. Y., and Y. H. Chu, 2001: Investigations of Blob-like Sporadic E Plasma Irregulari- 
ties Using the Chung-Li VHF Radar. J. Atmos. Solar-Terr. Phys., 63, 123-133.

Woodman, R. F., 1971: Inclination of the geomagnetic field measured by an incoherent scatter radar technique. J. Geophys. Res., 76, 178-184. 